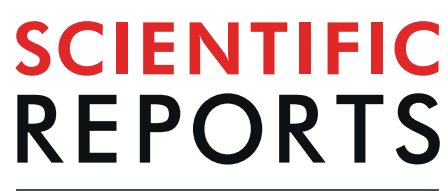

natureresearch

\title{
Proton pump inhibitors act synergistically with fluconazole against resistant Candida albicans
}

\begin{abstract}
Mengjiao Lu ${ }^{1,2}$, Haiying Yan ${ }^{3}$, Cuixiang Yú ${ }^{4}$, LeiYuan ${ }^{2} \&$ Shujuan Sun ${ }^{3 *}$
The incidence of resistant Candida isolates, especially Candida albicans, has increased continuously. To overcome the resistance, research on antifungal agent sensitizers has attracted considerable attention. Omeprazole and lansoprazole were found to inhibit the growth of sensitive $C$. albicans and hyphae formation in a high dose, respectively. This study aimed to determine the interactions of common clinically proton pump inhibitors (PPIs) and fluconazole both in vitro and in vivo and to further explore the possible mechanisms. In vitro, the tested PPIs all acted synergistically with fluconazole against both resistant $C$. albicans planktonic cells and biofilms preformed for $\leq 12 \mathrm{~h}$ with the minimum inhibitory concentration of fluconazole decreased from $>512 \mu \mathrm{g} / \mathrm{mL}$ to $1-4 \mu \mathrm{g} / \mathrm{mL}$. In vivo, PPIs plus fluconazole prolonged the survival rate of infected Galleria mellonella larvae by two-fold compared with that for the fluconazole monotherapy group and significantly reduced the tissue damage of infected larvae. Mechanism studies showed that PPIs significantly suppressed efflux pump activity, which is the common resistance mechanism of $C$. albicans, and significantly inhibited the virulence factors: phospholipase activity and morphology switching. These findings will provide new insights into antifungal agent discovery and potential approaches for the treatment of candidiasis caused by resistant $C$. albicans.
\end{abstract}

The incidence of invasive fungal infections has increased continuously, especially those caused by Candida species $^{1,2}$. Candida species can cause superficial infection of the skin, mouth, or mucous membranes and can also cause invasive infection, such as candidemia and biofilm-related infection ${ }^{3}$. In Candida infections, C. albicans is still the most commonly isolated strain. Data from the Prospective Antifungal Therapy Alliance registry showed that among the 7526 fungi isolated from 6807 invasive fungal infections, the isolation rate of Candida species was highest $(\mathrm{n}=5526,73.4 \%)$, and C. albicans accounted for $47.8 \%$ of its isolation rate ${ }^{4}$. Owing to its great efficacy and low toxicity, fluconazole (FLC) has been extensively used in clinical practice to prevent and treat candidiasis. However, along with the increased in frequency of infections and extensive use of FLC, drug-resistant strains have frequently emerged $^{5,6}$. To overcome fungal resistance, research on antifungal sensitizers has attracted considerable attention.

Proton pump inhibitors (PPIs) inhibit the $\mathrm{H}^{+} / \mathrm{K}^{+}$-ATPase in the cell membrane and have become the first choice in the treatment of acid-related diseases ${ }^{7,8}$. PPIs with a wide range of clinical applications include omeprazole (OME), lansoprazole (LAN), pantoprazole (PTP), rabeprazole (RAB), esomeprazole (ESO) and ilaprazole (ILA). OME was found to cure acute oesophageal necrosis and candidal oesophageal when it was combined with FLC in the clinic ${ }^{9-11}$. Studies on the antifungal activity of PPIs found that LAN and OME at a dose of $>600 \mu \mathrm{g} /$ $\mathrm{mL}$ could inhibit the growth of sensitive $C$. albicans and hyphae formation, respectively ${ }^{12,13}$. In addition, although some studies showed that PPIs combined with fluconazole in vitro have no synergistic effects against sensitive $C$. albicans $^{13-15}$, other studies found that BM2, a D-octapeptide inhibitor of the plasma membrane proton pump, enhanced the efficacy of FLC against resistant C. albicans and Candida dubliniensis ${ }^{16,17}$. However, no study has reported the interaction of commonly used PPIs and FLC against resistant C. albicans. In this study, we evaluated in vitro and in vivo interactions of PPIs combined with FLC against resistant C. albicans, and the underlying mechanism of the interactions of PPIs and FLC was further explored.

\footnotetext{
${ }^{1}$ Department of Pharmacy, Shandong Provincial Qianfoshan Hospital, Shandong University, Ji'nan, 250014, Shandong Province, P.R. China. ${ }^{2}$ Department of Pharmacy, Tianjin Baodi Hospital, Baodi Clinical College of Tianjin Medical University, Tianjin, 301800, P.R. China. ${ }^{3}$ Department of Pharmacy, Shandong Provincial Qianfoshan Hospital, the First Hospital Affiliated with Shandong First Medical University, Ji'nan, 250014, Shandong Province, P.R. China. ${ }^{4}$ Respiration Medicine, Shandong Provincial Qianfoshan Hospital, the First Hospital Affiliated with Shandong First Medical University, Ji'nan, 250014, Shandong Province, P.R. China. *email: sunshujuan888@163.com
} 


\begin{tabular}{|c|c|c|c|c|c|c|c|c|c|c|}
\hline \multirow[b]{3}{*}{ PPIs $^{\mathbf{a}}$} & \multirow[b]{3}{*}{ Strains ${ }^{b}$} & \multicolumn{4}{|c|}{$\operatorname{MIC}_{80}(\mu \mathrm{g} / \mathrm{mL})^{\mathrm{c}}$} & \multicolumn{2}{|c|}{ FICI model } & \multicolumn{3}{|c|}{$\Delta E$ model } \\
\hline & & \multicolumn{2}{|l|}{ Alone } & \multicolumn{2}{|c|}{ Combined } & \multirow[b]{2}{*}{$\mathbf{F I C I}^{\mathbf{c}}$} & \multirow[b]{2}{*}{$\mathrm{IN}^{\mathrm{a}}$} & \multirow{2}{*}{$\begin{array}{l}\sum S Y N \\
(\%) c\end{array}$} & \multirow{2}{*}{$\begin{array}{l}\underset{\text { SANT }}{(\%) \mathrm{c}} \\
\end{array}$} & \multirow[b]{2}{*}{$\mathrm{IN}^{\mathrm{a}}$} \\
\hline & & PPIs & FLC & PPIs & FLC & & & & & \\
\hline \multirow{6}{*}{ OME } & CA10 & $>512$ & $>512$ & 32 & 0.5 & 0.06 & SYN & 1469.95 & -5.31 & SYN \\
\hline & CA16 & $>512$ & $>512$ & 32 & 0.5 & 0.06 & SYN & 1663.1 & -18.05 & SYN \\
\hline & CA103 & $>512$ & $>512$ & 32 & 1 & 0.06 & SYN & 1196.35 & -9.63 & SYN \\
\hline & CA137 & $>512$ & $>512$ & 32 & 1 & 0.06 & SYN & 1089.68 & -9.77 & SYN \\
\hline & CA632 & $>512$ & $>512$ & 32 & 1 & 0.06 & SYN & 1100.53 & -3.94 & SYN \\
\hline & CA20003 & $>512$ & $>512$ & 32 & 0.5 & 0.06 & SYN & 1101.23 & -3.92 & SYN \\
\hline \multirow{6}{*}{ LAN } & CA10 & $>512$ & $>512$ & 32 & 1 & 0.06 & SYN & 1244.77 & -0.61 & SYN \\
\hline & CA16 & $>512$ & $>512$ & 32 & 1 & 0.06 & SYN & 1437.05 & -66.34 & SYN \\
\hline & CA103 & $>512$ & $>512$ & 32 & 1 & 0.06 & SYN & 1079.33 & -15.94 & SYN \\
\hline & CA137 & $>512$ & $>512$ & 16 & 1 & 0.03 & SYN & 816.71 & -7.27 & SYN \\
\hline & CA632 & $>512$ & $>512$ & 32 & 1 & 0.06 & SYN & 1249.21 & -60.38 & SYN \\
\hline & CA20003 & $>512$ & $>512$ & 32 & 1 & 0.06 & SYN & 1103.44 & -44.94 & SYN \\
\hline \multirow{6}{*}{ PTP } & CA10 & $>512$ & $>512$ & 64 & 1 & 0.13 & SYN & 1341.94 & -8.84 & SYN \\
\hline & CA16 & $>512$ & $>512$ & 64 & 1 & 0.13 & SYN & 1653.07 & -24.73 & SYN \\
\hline & CA103 & $>512$ & $>512$ & 32 & 1 & 0.06 & SYN & 1106.52 & -95.84 & SYN \\
\hline & CA137 & $>512$ & $>512$ & 32 & 1 & 0.06 & SYN & 823.01 & -64.51 & SYN \\
\hline & CA632 & $>512$ & $>512$ & 64 & 1 & 0.13 & SYN & 1177.14 & -63.02 & SYN \\
\hline & CA20003 & $>512$ & $>512$ & 64 & 1 & 0.13 & SYN & 1057.08 & -53.32 & SYN \\
\hline \multirow{6}{*}{ RAB } & CA10 & 256 & $>512$ & 16 & 1 & 0.06 & SYN & 1149.42 & -27.13 & SYN \\
\hline & CA16 & 256 & $>512$ & 16 & 1 & 0.06 & SYN & 1157.63 & -9.11 & SYN \\
\hline & CA103 & 256 & $>512$ & 32 & 2 & 0.13 & SYN & 1044.78 & -79.34 & SYN \\
\hline & CA137 & 256 & $>512$ & 16 & 2 & 0.07 & SYN & 876.45 & -74.15 & SYN \\
\hline & CA632 & 512 & $>512$ & 16 & 4 & 0.04 & SYN & 1168.12 & -163.45 & SYN \\
\hline & CA20003 & 128 & $>512$ & 32 & 2 & 0.25 & SYN & 1235.58 & -130.41 & SYN \\
\hline \multirow{6}{*}{ ESO } & CA10 & $>512$ & $>512$ & 32 & 1 & 0.06 & SYN & 1334.34 & -18.4 & SYN \\
\hline & CA16 & $>512$ & $>512$ & 32 & 1 & 0.06 & SYN & 1316.71 & -1.07 & SYN \\
\hline & CA103 & $>512$ & $>512$ & 32 & 1 & 0.06 & SYN & 1178.25 & -83.86 & SYN \\
\hline & CA137 & $>512$ & $>512$ & 32 & 1 & 0.06 & SYN & 1146.03 & -49.13 & SYN \\
\hline & CA632 & $>512$ & $>512$ & 32 & 1 & 0.06 & SYN & 1062.42 & -18.3 & SYN \\
\hline & CA20003 & $>512$ & $>512$ & 32 & 1 & 0.06 & SYN & 1086.97 & -26.40 & SYN \\
\hline \multirow{6}{*}{ ILA } & CA10 & $>256$ & $>512$ & 8 & 1 & 0.03 & SYN & 1072.23 & -5.44 & SYN \\
\hline & CA16 & $>256$ & $>512$ & 8 & 1 & 0.03 & SYN & 1201.75 & -10.62 & SYN \\
\hline & CA103 & $>256$ & $>512$ & 16 & 1 & 0.06 & SYN & 944.53 & -95.54 & SYN \\
\hline & CA137 & $>256$ & $>512$ & 8 & 1 & 0.03 & SYN & 1045.04 & -7.57 & SYN \\
\hline & CA632 & $>256$ & $>512$ & 8 & 2 & 0.04 & SYN & 953.47 & -151.16 & SYN \\
\hline & CA20003 & $>256$ & $>512$ & 8 & 1 & 0.03 & SYN & 1306.81 & -47.30 & SYN \\
\hline
\end{tabular}

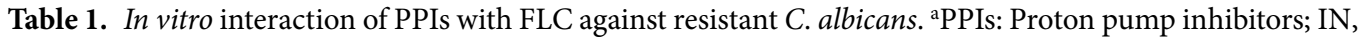
interpretation; SYN, Synergism; OME, omeprazole; LAN, Lansprazole; PTP, Pantoprazole; RAB, Rabeprazole; ESO, Esomeprazole; ILA, Ilaprazole; ${ }^{\mathrm{b}} \mathrm{CA}$, Candida albicans; ${ }^{\mathrm{c}} \mathrm{The} \mathrm{MIC}_{80} \mathrm{MIC}$ was defined as the lowest concentration showing $80 \%$ growth inhibition; FICI, fractional inhibitory concentration index; $\Sigma$ SYN and $\Sigma A N T$ were the sums of the percentages of all statistically significant synergistic and antagonistic interactions; $\mathrm{MIC}_{80}$ values, FICIs, $\Sigma$ SYN and $\Sigma$ ANT are the median of three independent experiments.

In the present study, the in vitro antifungal activity of PPIs alone or combined with FLC was determined by the microdilution method, and an XTT assay was conducted to evaluate the antibiofilm effects of the drug combination. In addition, the in vivo interaction of the drug combination was evaluated by the establishment of a $G$. mellonella larvae infection model. Of note, with OME and RAB as representative PPI drugs, synergistic mechanisms were evaluated by assessing extracellular phospholipase activity, morphology switching and the efflux pump activity.

\section{Results}

PPIs acted synergistically with FLC against resistant $C$. albicans in vitro. The minimal inhibitory concentrations (MICs) of PPIs and FLC against resistant C. albicans are listed in Table 1. The MIC of FLC was all $>512 \mu \mathrm{g} / \mathrm{mL}$ for all tested C. albicans strains, indicating strong resistance of these C. albicans strains. The MICs of RAB, ILA and the others were $128-512 \mu \mathrm{g} / \mathrm{mL},>256 \mu \mathrm{g} / \mathrm{mL}$ and $>512 \mu \mathrm{g} / \mathrm{mL}$, respectively, showing that RAB possessed a weak intrinsic antifungal activity and a very limited intrinsic efficacy for the other PPIs. However, when used in combination with FLC, PPIs could significantly decrease the MICs of FLC from $>512 \mu \mathrm{g} / \mathrm{mL}$ to 


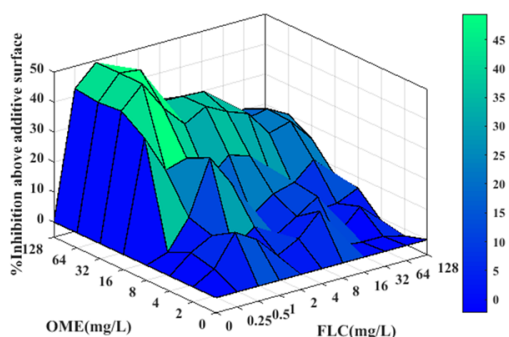

A

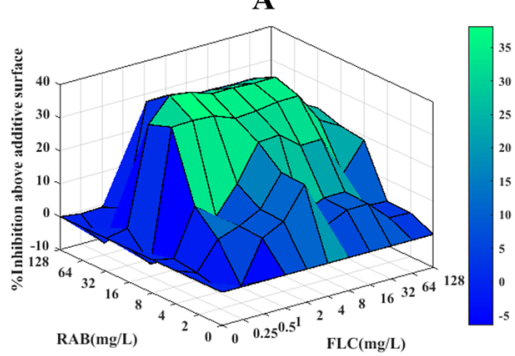

D

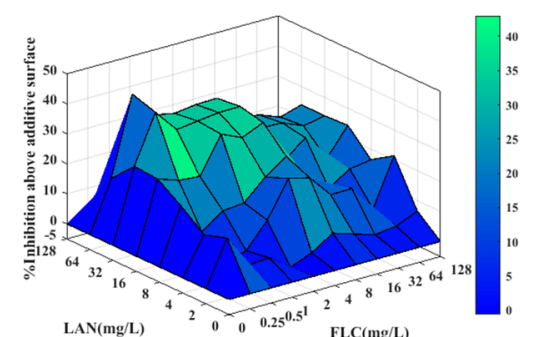

B

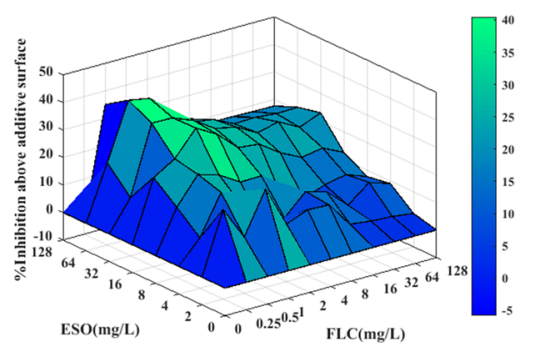

$\mathbf{E}$

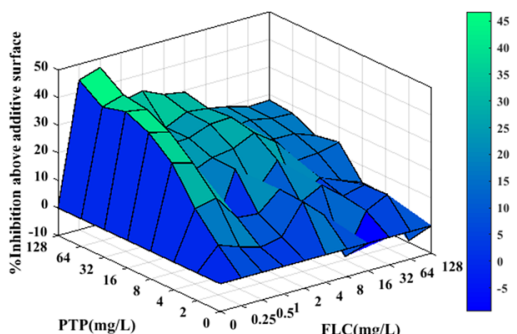

C

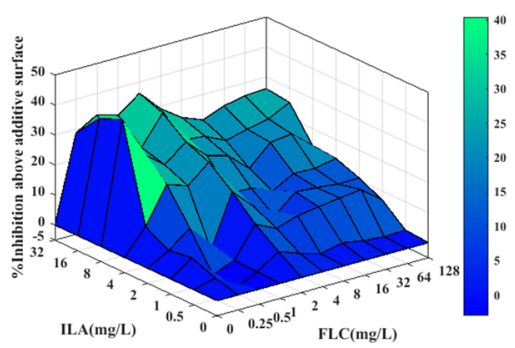

F

Figure 1. Three-dimensional model of PPIs combined with FLC against CA10 in vitro. (A-F) show the threedimensional model of OME, LAN, PTP, RAB, ESO and ILA combined with FLC, respectively. The $\Delta E$ values are depicted on the $z$-axis, and the peaks above the 0 plane indicate synergistic combinations, whereas the peaks below the 0 plane indicate antagonistic combinations.

$0.5-4 \mu \mathrm{g} / \mathrm{mL}$, indicating a significantly increased sensitivity of resistant C. albicans to FLC caused by PPIs. Of these six PPIs, when the MIC of FLC was decreased to $\leq 2 \mu \mathrm{g} / \mathrm{mL}$, the concentrations of PPIs required were $8 \mu \mathrm{g} /$ $\mathrm{mL}$ for ILA, $16-32 \mu \mathrm{g} / \mathrm{mL}$ for RAB, $16-32 \mu \mathrm{g} / \mathrm{mL}$ for LAN, $32 \mu \mathrm{g} / \mathrm{mL}$ for OME, $32 \mu \mathrm{g} / \mathrm{mL}$ for ESO and $32-64 \mu \mathrm{g} /$ $\mathrm{mL}$ for PTP. Moreover, the FICI values obtained from the FICI model were 0.06 for OME and ESO, $0.03-0.06$ for LAN and ILA, $0.06-0.13$ for PTP and $0.04-0.25$ for RAB. The FICI values were all $<0.5$, showing a strong synergism induced by PPIs plus FLC. Additionally, this synergistic effect was demonstrated by another evaluation model (Table 1, Fig. 1), with the $\Sigma$ SYN values all $>800 \%$, far more than $200 \%$, indicating that PPIs in combination with FLC exerted synergistic inhibitory effects on the growth of resistant C. albicans.

PPIs synergized with FLC against preformed biofilms of CA10. The sessile minimum inhibitory concentrations (sMIC) of PPIs and FLC against a resistant C. albicans strain (CA10) are listed in Table 2, and the data were analysed by the FICI model. For the biofilms preformed over 4,8 and $12 \mathrm{~h}$, the sMIC $_{80}$ of FLC decreased significantly from $>1024 \mu \mathrm{g} / \mathrm{mL}$ to $1-4 \mu \mathrm{g} / \mathrm{mL}$, with the FICI values $0.06-0.13$ for OME and RAB and $0.06-0.25$ for LAN, PTP, ESO and ILA. The FICI values were all $<0.5$, showing strong synergistic antibiofilm effects induced by PPIs and FLC. When combined with PPIs against biofilms preformed over $24 \mathrm{~h}$, there was almost no change in the $\mathrm{SMIC}_{80}$ of FLC compared with that of FLC alone, indicating an indifferent interaction between PPIs and FLC.

PPIs enhanced the efficacy of FLC against resistant $C$. albicans in vivo. In an in vivo experiment, 20 randomly chosen larvae in each group were injected with a C. albicans suspension, and after $2 \mathrm{~h}$ of infection, the larvae were treated with drugs. Regarding the survival rate of G. mellonella larvae (Fig. 2), 25\% of the larvae in the control group survived until the end of observation period. With the monotherapy of FLC and PPIs, the survival rates of larvae were $20-35 \%$, similar to that of the control group, indicating no significant antifungal effect of drug monotherapy on the larvae. Notably, PPIs combined with FLC kept the larvae free from C. albicans infections and resulted in 70-85\% survival of the larvae over a 4-day infection. More specifically, the survival rates of the drug combination groups were $85 \%$ for OME and LAN, $80 \%$ for PTP, $75 \%$ for ESO, and 70\% for RAB and ILA, demonstrating that the combination of PPIs and FLC significantly increased the survival rates of infected larvae $(P<0.05)$.

Regarding observation of histological sections (Fig. 3), integrated and dense tissue was observed in the blank group with no black mass and uniform staining. In other groups, the infected tissues showed black lumps after PAS staining, and the lumps contained yeast cells and hyphae. More specifically, black lumps in the FLC-monotherapy group and PPI-monotherapy groups as well as the control group were numerous and large, while those in the combination treatment groups were obviously much fewer and smaller. These observations suggested that compared with the FLC monotherapy, PPIs combined with FLC significantly reduced the damage of the resistant C. albicans to the larvae.

PPIs plus FLC synergistically suppressed the morphology switching of CA10. The hyphae growth of CA10 after the drug treatments was observed to evaluate the effect of the drug combination on the morphologic transformation of the resistant C. albicans. The results showed (Fig. 4) that the length of hyphae in the FLC and $\mathrm{RAB}$ monotherapy groups was much shorter than that in the control group and the OME monotherapy group, indicating a weak inhibitory effect induced by FLC and RAB but not by OME. Importantly, compared with that 


\begin{tabular}{|c|c|c|c|c|c|c|c|}
\hline \multirow[b]{3}{*}{ PPIs ${ }^{\mathrm{a}}$} & \multirow{3}{*}{$\begin{array}{l}\text { Time } \\
(\mathbf{h})^{\mathbf{b}}\end{array}$} & \multicolumn{4}{|c|}{ sMIC $_{80}$ of drugs $(\mu \mathrm{g} / \mathrm{mL})^{\mathrm{c}}$} & \multirow[b]{3}{*}{ FICI $^{\mathfrak{c}}$} & \multirow[b]{3}{*}{$\mathrm{IN}^{\mathrm{a}}$} \\
\hline & & \multicolumn{2}{|l|}{ Alone } & \multicolumn{2}{|c|}{ Combined } & & \\
\hline & & PPIs & FLC & PPIs & FLC & & \\
\hline \multirow{4}{*}{ OME } & 4 & $>1024$ & $>1024$ & 64 & 1 & 0.06 & SYN \\
\hline & 8 & $>1024$ & $>1024$ & 64 & 2 & 0.06 & SYN \\
\hline & 12 & $>1024$ & $>1024$ & 128 & 4 & 0.13 & SYN \\
\hline & 24 & $>1024$ & $>1024$ & $>1024$ & $>1024$ & 2 & IND \\
\hline \multirow{4}{*}{ LAN } & 4 & $>512$ & $>1024$ & 32 & 2 & 0.06 & SYN \\
\hline & 8 & $>512$ & $>1024$ & 64 & 1 & 0.13 & SYN \\
\hline & 12 & $>512$ & $>1024$ & 128 & 2 & 0.25 & SYN \\
\hline & 24 & $>512$ & $>1024$ & $>512$ & $>1024$ & 2 & IND \\
\hline \multirow{4}{*}{ PTP } & 4 & $>1024$ & $>1024$ & 64 & 2 & 0.06 & SYN \\
\hline & 8 & $>1024$ & $>1024$ & 128 & 2 & 0.13 & SYN \\
\hline & 12 & $>1024$ & $>1024$ & 256 & 2 & 0.25 & SYN \\
\hline & 24 & $>1024$ & $>1024$ & $>1024$ & $>1024$ & 2 & IND \\
\hline \multirow{4}{*}{ RAB } & 4 & $>1024$ & $>1024$ & 32 & 1 & 0.03 & SYN \\
\hline & 8 & $>1024$ & $>1024$ & 64 & 1 & 0.06 & SYN \\
\hline & 12 & $>1024$ & $>1024$ & 128 & 2 & 0.13 & SYN \\
\hline & 24 & $>1024$ & $>1024$ & $>1024$ & $>1024$ & 2 & IND \\
\hline \multirow{4}{*}{ ESO } & 4 & $>1024$ & $>1024$ & 64 & 1 & 0.06 & SYN \\
\hline & 8 & $>1024$ & $>1024$ & 64 & 2 & 0.06 & SYN \\
\hline & 12 & $>1024$ & $>1024$ & 256 & 4 & 0.25 & SYN \\
\hline & 24 & $>1024$ & $>1024$ & $>1024$ & $>1024$ & 2 & IND \\
\hline \multirow{4}{*}{ ILA } & 4 & $>256$ & $>1024$ & 16 & 1 & 0.06 & SYN \\
\hline & 8 & $>256$ & $>1024$ & 32 & 2 & 0.13 & SYN \\
\hline & 12 & $>256$ & $>1024$ & 64 & 1 & 0.25 & SYN \\
\hline & 24 & $>256$ & $>1024$ & $>256$ & $>1024$ & 2 & IND \\
\hline
\end{tabular}

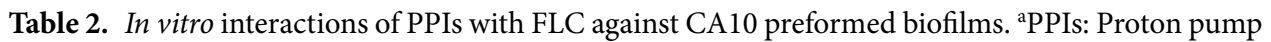
inhibitors; IN, interpretation; IND, Indifference; SYN, Synergism; OME, omeprazole; LAN, Lansprazole; PTP, Pantoprazole; RAB, Rabeprazole; ESO, Esomeprazole; ILA, Ilaprazole; ${ }^{\text {b}}$ Time, incubation period of preformed biofilm; 'The $\mathrm{SMIC}_{80} \mathrm{MIC}^{\mathrm{C}}$ was defined as the lowest concentration showing $80 \%$ biofilm metabolic activity inhibition; FICI, fractional inhibitory concentration index; $\mathrm{sMIC}_{80}$ values and FICIs are the median of three independent experiments.

in the control group and the drug monotherapy groups, the length of hyphae in the drug combination groups, namely OME + FLC and RAB + FLC, was visibly shorter. Therefore, the drug combination could inhibit filamentous growth of drug-resistant C. albicans cells, and the inhibitory effects were higher than that of FLC alone.

PPIs plus FLC synergistically inhibited the phospholipase activity of CA10. The $\mathrm{P}_{Z}$ value was calculated as $\mathrm{P}_{Z}=$ the diameter of colony/the diameter (colony + precipitation zone) to evaluate the phospholipase activity of $C$. albicans, and the higher the $\mathrm{P}_{Z}$ value was, the lower the phospholipase activity. In this study, very high phospholipase activity was observed in the control group and drug monotherapy group with $\mathrm{P}_{Z}$ values of $0.64-0.66$ (Table 3 ). For the combination groups, the $\mathrm{P}_{\mathrm{Z}}$ values were $0.87 \pm 0.01$ for OME plus FLC and $0.84 \pm 0.01$ for RAB plus FLC, showing a significantly lower phospholipase activity than that in other groups $(P<$ 0.0001). These data indicated that PPIs combined with FLC could synergistically decrease the phospholipase activity of resistant C. albicans.

PPIs inhibited the efflux pump activity of CA10. The fluorescent dye rhodamine $6 \mathrm{G}$ (Rh6G) and FLC both are substrates of drug transporters in C. albicans. Therefore, in this assay, we used Rh6G as a tracer of FLC to detect the intracellular FLC concentration. As shown in Fig. 5, the initial values of the MFI in the control group and the PPI-treated groups were almost the same, and the MFI showed a decreasing trend over time. Within $120 \mathrm{~min}$, there was no difference between the MFI of control group and the PPI-treated groups. However, after $240 \mathrm{~min}$, the MFI of the control group decreased significantly, while that of the PPI-treated groups decreased slightly. This result indicated that the addition of PPIs could inhibit the efflux pump activity of resistant C. albicans.

\section{Discussion}

In recent years, the resistance of C. albicans to current antifungal agents has emerged frequently, and to solve this problem, research on sensitizers to existing antifungal agents or new antifungal drugs has received wide attention. Numerous studies on sensitizers to antifungal agents found that antibacterials ${ }^{18}$, calcium channel blockers ${ }^{19}$, phytocompounds ${ }^{20}$, etc., could enhance the sensitivity of resistant C. albicans to antifungals. 

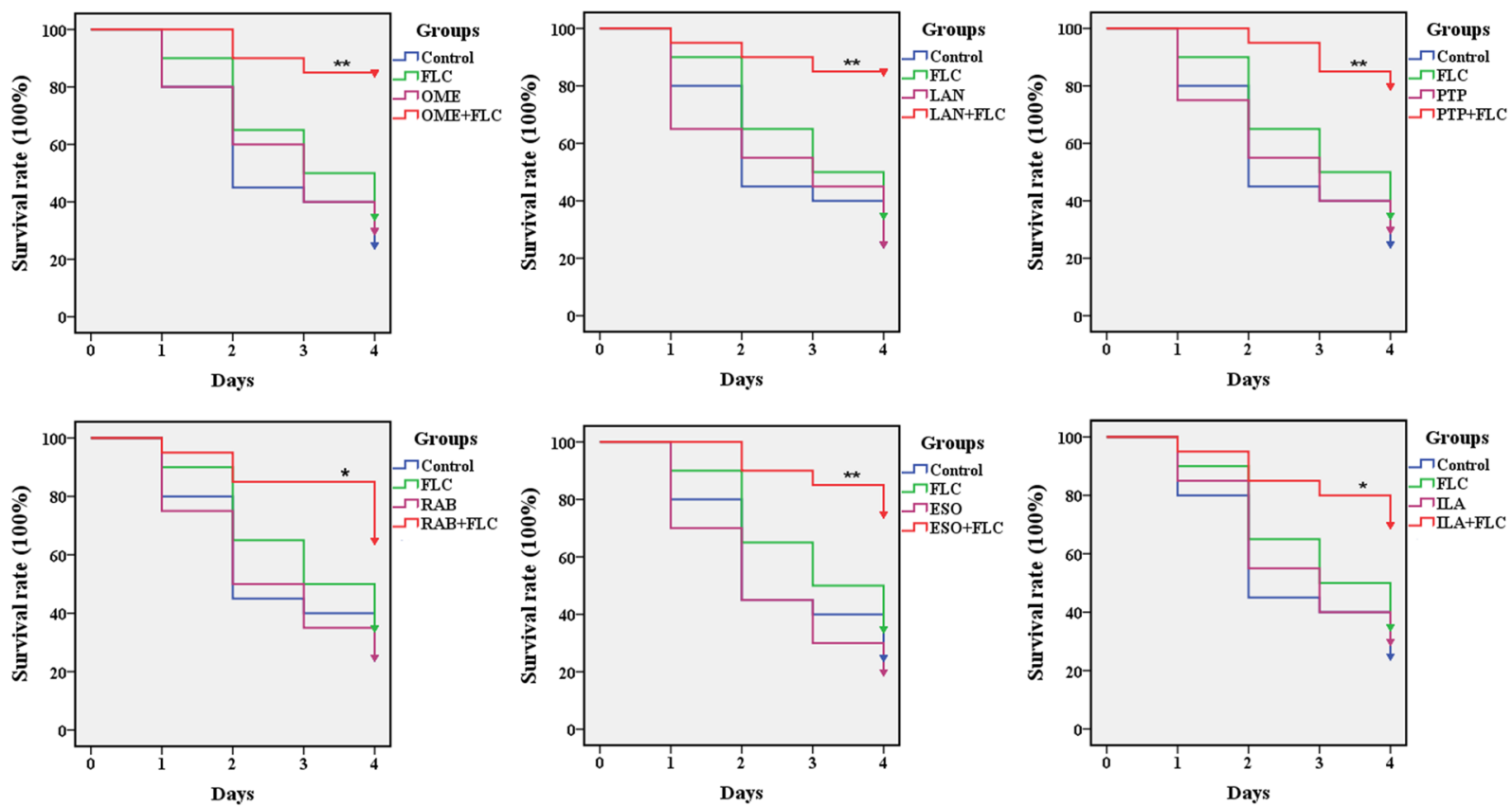

Figure 2. Survival rate of infected G. mellonella larvae treated with different drugs. After infection with CA10 $\left(5 \times 10^{8} \mathrm{CFU} / \mathrm{mL}\right)$, larvae were treated with PBS, FLC $(160 \mu \mathrm{g} / \mathrm{mL})$, OME $(80 \mu \mathrm{g} / \mathrm{mL}), \mathrm{LAN}(80 \mu \mathrm{g} / \mathrm{mL})$, PTP $(160 \mu \mathrm{g} / \mathrm{mL}), \mathrm{RAB}(40 \mu \mathrm{g} / \mathrm{mL})$, ESO $(80 \mu \mathrm{g} / \mathrm{mL})$, ILA $(40 \mu \mathrm{g} / \mathrm{mL})$ or PPIs plus FLC $(160 \mu \mathrm{g} / \mathrm{mL})$. The log-rank test was performed, and results were compared with the FLC-treated group; $* P<0.05$, $* * P<0.01$.

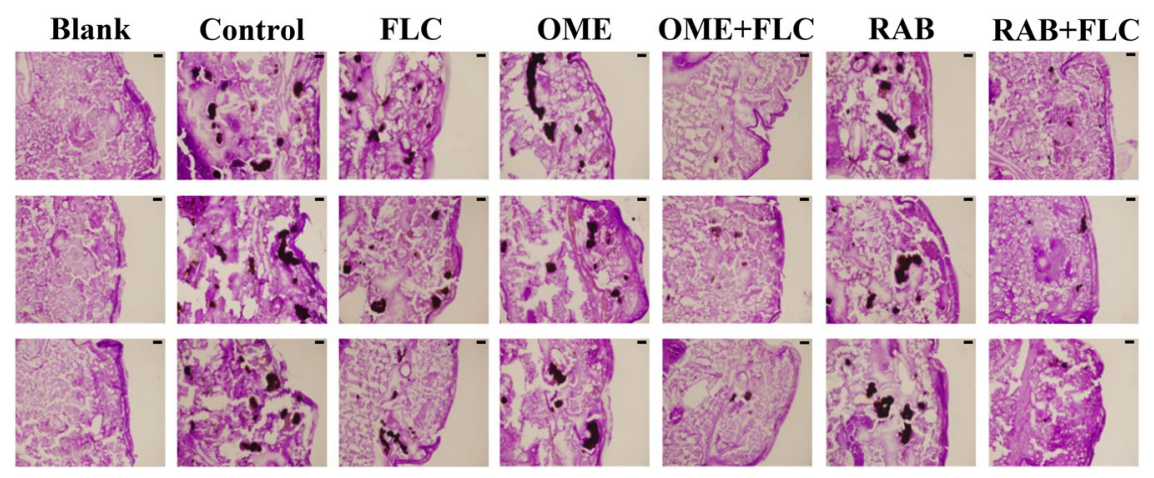

Figure 3. Histopathology of infected G. mellonella larvae treated with different drugs. After infection with CA10 $\left(5 \times 10^{8} \mathrm{CFU} / \mathrm{mL}\right)$, larvae were treated with PBS, FLC $(160 \mu \mathrm{g} / \mathrm{mL}), \mathrm{OME}(80 \mu \mathrm{g} / \mathrm{mL}), \mathrm{RAB}(40 \mu \mathrm{g} / \mathrm{mL})$, OME $(80 \mu \mathrm{g} / \mathrm{mL})$ plus FLC $(160 \mu \mathrm{g} / \mathrm{mL})$ or RAB $(40 \mu \mathrm{g} / \mathrm{mL})$ plus FLC $(160 \mu \mathrm{g} / \mathrm{mL})$. The larvae of the blank groups were not treated with yeast or any drugs. Tissue sections were observed at a $4.2 \times 10$ multiplier, with a scale of $20 \mu \mathrm{m}$.

As the most potent acid-suppressing drugs, PPIs are considered as the standard treatment for acid-related diseases. Although acid suppression therapy has been reported as a risk factor for Candida oesophagitis ${ }^{21}$, PPIs represent an opportunity for joint applications with FLC for Candida oesophagitis in the clinic. For example, Hasosah, M. Y. et al. and Liang, M. et al. reported that immunocompetent patients presenting with Candida oesophagitis were successfully cured by OME combined with $\mathrm{FLC}^{22,23}$. Studies on the antifungal interaction of PPIs and FLC have also been conducted previously, and some studies showed antagonistic effects against susceptible C. albicans isolates ${ }^{13,14}$. However, there was no report about whether PPIs could increase the sensitivity of resistant C. albicans to FLC. Based on these studies, we tested the interaction of PPIs combined with FLC against both susceptible and resistant $C$. albicans strains. In a preliminary experiment, we observed a strange phenomenon for all the drug combinations against the susceptible strain. The combination of FLC and RAB serves as an example to clarify this phenomenon. As shown in Table S1, compared with $0.25 \mu \mathrm{g} / \mathrm{mL}$ FLC alone, the addition of 4-32 $\mu \mathrm{g} / \mathrm{mL}$ RAB showed a stronger inhibitory effect on the C. albicans susceptible strain CA4; however, a higher concentration of RAB $(64-128 \mu \mathrm{g} / \mathrm{mL})$ resulted in a weaker inhibitory effect. To explain this phenomenon, many experiments need to be done. Therefore, we focused only on the resistant strain in this study. More importantly, 


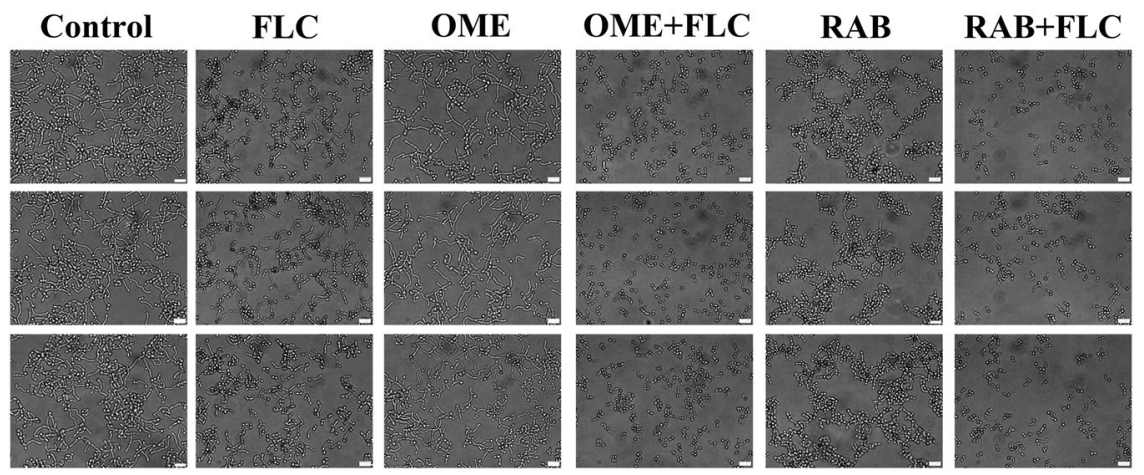

Figure 4. Effects of PPIs combined with FLC on the morphologic transformation of resistant C. albicans. A yeast suspension $\left(5 \times 10^{5} \mathrm{CFU} / \mathrm{mL}\right)$ was incubated in RPMI-1640 medium with PBS, FLC $(8 \mu \mathrm{g} / \mathrm{mL})$, OME $(128 \mu \mathrm{g} / \mathrm{mL})$, OME $(128 \mu \mathrm{g} / \mathrm{mL})$ plus FLC $(8 \mu \mathrm{g} / \mathrm{mL}), \mathrm{RAB}(64 \mu \mathrm{g} / \mathrm{mL})$ or RAB $(64 \mu \mathrm{g} / \mathrm{mL})$ plus FLC $(8 \mu \mathrm{g} / \mathrm{mL})$. After an incubation of $5 \mathrm{~h}$ at $35^{\circ} \mathrm{C}$, cells were observed under an inverted microscope at a $40 \times 10$ multiplier, and the scale in the figure is $20 \mu \mathrm{m}$.
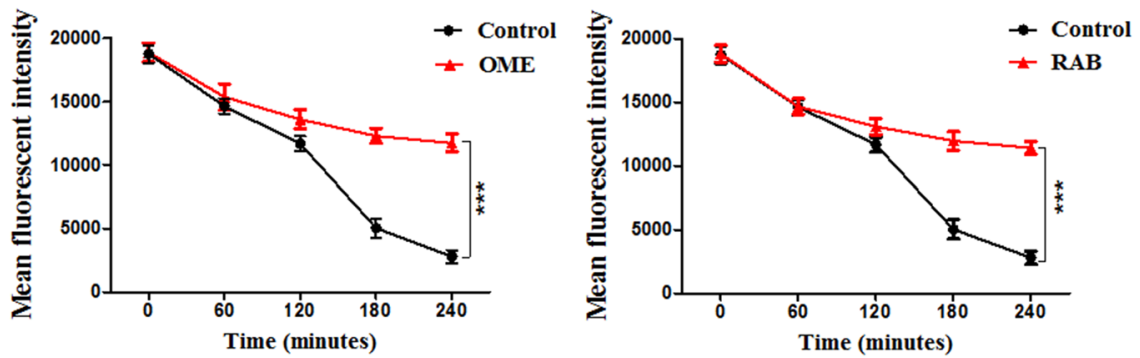

Figure 5. Inhibitory effects of PPIs on the efflux of R6G in resistant C. albicans. Fluorescent intensity was detected after the treatment with OME $(128 \mu \mathrm{g} / \mathrm{mL})$ and RAB $(64 \mu \mathrm{g} / \mathrm{mL})$ over $240 \mathrm{~min}$. The statistical significance was determined by Student's $t$-test and compared with the control group; $* * * P<0.001$.

\begin{tabular}{|l|l|l|}
\hline Drugs $^{\mathbf{a}}$ & $\mathbf{P}_{\mathbf{z}}$ value $\pm \mathbf{S D}^{\mathbf{b}}$ & $\begin{array}{l}\text { Phospholipase } \\
\text { activity }\end{array}$ \\
\hline No drug & $0.64 \pm 0.02$ & Very high \\
\hline FLC & $0.66 \pm 0.02^{\text {n.s }}$ & Very high \\
\hline OME & $0.65 \pm 0.01^{\text {n.s }}$ & Very high \\
\hline RAB & $0.66 \pm 0.01^{\text {n.s }}$ & Very high \\
\hline OME + FLC & $0.87 \pm 0.01^{* * * *}$ & Low \\
\hline RAB + FLC & $0.84 \pm 0.01^{* * * *}$ & Low \\
\hline
\end{tabular}

Table 3. Phospholipase activity of resistant C. albicans (CA10) treated with drugs. ${ }^{\text {aFLC }}$, fluconazole $(1 \mu \mathrm{g} / \mathrm{mL})$; OME, omeprazole $(32 \mu \mathrm{g} / \mathrm{mL})$; RAB, rabeprazole $(16 \mu \mathrm{g} / \mathrm{mL}) ;{ }^{b} P_{z}$ values were the median of three independent experiments; $\mathrm{Pz} \leq 0.69$, very high phospholipase activity, $\mathrm{Pz}=0.70-0.79$, high activity; $\mathrm{Pz}=0.80-0.89$, low activity; $\mathrm{Pz}=0.90-0.99$, very low activity; $\mathrm{Pz}=1$, negative activity; $\mathrm{SD}$, standard deviation; Compared with the control group, ${ }^{\text {n.s }} P>0.05$; Compared with the control group and drug monotherapy groups; $* * * * P<0.0001$.

Monk, B. C. et al. and Hayama, K. et al. found that an inhibitor of the plasma membrane proton pump (BM2) could enhance the efficacy of FLC against resistant C. albicans and C. dubliniensis ${ }^{16}$. Inspired by the synergism of BM2 with FLC, we evaluated the interaction of PPIs combined with FLC against resistant $C$. albicans and further explored the underlying mechanisms.

In vitro, we found that PPIs exerted weak anticandidal effects with MICs $\geq 128 \mu \mathrm{g} / \mathrm{mL}$, which was in accordance with the studies of Biswas, S. K. et al. and Liu, N et al. ${ }^{12,13}$. More importantly, PPIs all acted synergistically with FLC against six tested resistant $C$. albicans isolates, as interpreted by the FICI and $\Delta E$ model. These six PPIs decreased the $\mathrm{MIC}_{80}$ of FLC from $>512 \mu \mathrm{g} / \mathrm{mL}$ to $0.5-4 \mu \mathrm{g} / \mathrm{mL}$. Among them, OME reduced the MICs of FLC to a maximum extent, while RAB reduced the MICs of FLC to a minimum extent. Additionally, when the MIC of FLC was decreased to the same concentration, the minimum concentration of proton pump inhibitors required is $8 \mu \mathrm{g} / \mathrm{mL}$ for ILA, and the maximum is $32-64 \mu \mathrm{g} / \mathrm{mL}$ for PTP, demonstrating that different PPIs resulted in different enhanced efficacy of FLC.

Biofilm formation has been shown to be related with drug resistance of C. albicans ${ }^{24}$. In clinic, biofilm-related infections are difficult to treat due to their trend to be chronic and easy recurrence. Here, the sMIC $_{80}$ of FLC for 
resistant C. albicans biofilms preformed for $\leq 12 \mathrm{~h}$ was decreased from $>1024 \mu \mathrm{g} / \mathrm{mL}$ to $1-4 \mu \mathrm{g} / \mathrm{mL}$ by the presence of PPIs, indicating enhancement of the antibiofilm effect of FLC by PPIs. In addition, different PPIs resulted in different enhanced efficacy of FLC. With prolonged preformation time, the biofilm was more mature, and the synergistic effect was weaker. The biofilm preformed for $24 \mathrm{~h}$ was much more mature and its structure was more complex. Therefore, no obvious inhibitory action induced by the drug combinations was observed. Studies on the antibiofilm effect of drugs also showed that drugs are less effective in mature C. albicans biofilms than in early-stage biofilms and even not effective in mature biofilms ${ }^{25,26}$. Our findings demonstrated a potential use of the drug combination in prevention or early treatment of biofilm-related diseases.

The G. mellonella larva is a type of infection model that has been used to study the efficacy or toxicity of drugs as well as the virulence of pathogens ${ }^{27,28}$. Compared with mammal host models, this infection model can provide a more rapid evaluation of the virulence of pathogens and the in vivo efficacy or toxicity of agents, with significant economic and ethical advantages ${ }^{29,30}$. To primarily assess the combined effects of PPIs and FLC in vivo, we used this model to determine survival rates of the larvae infected with CA10. The data obtained showed that the survival rates of the larvae treated with PPIs + FLC were significantly higher than those of the FLC monotherapy group and other groups, demonstrating that the tested PPIs could significantly increase the in vivo efficacy of FLC against resistant C. albicans. Histopathological study of G. mellonella larvae was carried out to further assess the combined effects of PPIs plus FLC in vivo. In this study, the tissue infected with CA10 was stained as black lumps and was much more fragmentary than that of the blank group. This result indicated that the resistant C. albicans CA10 could cause serious damage to the tissues of the larvae. In addition, fewer and smaller black lumps were observed in the drug combination group than in the control group and the drug-monotherapy groups, demonstrating that at the experimental concentration, the drug combinations could suppress the damage caused by resistant C. albicans to the larvae. Taken together, these data show that PPIs could significantly enhance the in vivo efficacy of FLC against resistant C. albicans, which was in accordance with the in vitro results.

C. albicans can switch among different morphological phenotypes, and the morphology switching is a primary virulence factor ${ }^{31,32}$. Notably, this switch has been proven to be involved in the pathogenicity and biofilms formation of C. albicans ${ }^{33,34}$. Here, we found that RAB possessed a weak inhibitory effect on the morphology switching of both CA10 and a susceptible C. albicans strain (CA4) (Fig. S1). More importantly, compared with FLC monotherapy, PPIs combined with FLC possessed a stronger inhibitory effect on hyphae formation. This finding demonstrated that the inhibition of the morphology switching might be a mechanism of the synergistic antifungal effect induced by PPIs combined with FLC. In addition, LAN was also found to possess a weak inhibitory effect on the hyphae formation of CA4 (Fig. S2), and this finding was coincident with earlier research, which reported the inhibition of hyphal growth of $C$. albicans by LAN.

Phospholipase, as one of the most important hydrolase of C. albicans, is another important virulence factor in C. albicans infections ${ }^{35,36}$. Ying et al. found that the phospholipase B1 mRNA and protein expression of resistant strains was higher than that of susceptible strains ${ }^{37}$. In addition, C. albicans phospholipase D1 has been proven to play a role in promoting the transformation of yeast to mycelium ${ }^{38}$. These studies demonstrated that the phospholipase activity of $C$. albicans might be related to the resistance and morphology switching. In the present study, the phospholipase activities of C. albicans in the PPIs plus FLC groups were significantly reduced, with $\mathrm{P}_{\mathrm{z}}$ values higher than 0.80 . However, drug combinations at the same concentrations could not decrease the phospholipase activity of a susceptible C. albicans (CA4) (Table S3). Inspired by the Ying et al. study ${ }^{37}$, we determined that the synergism of PPIs and FLC against resistant C. albicans might be related to phospholipase B1, and this observation needs further study.

As one of the most common resistance mechanisms in C. albicans, overexpression of efflux pumps has become a research hotspot for Candida resistance ${ }^{39,40}$. The present study found that compared with the control group, the addition of PPIs significantly suppressed efflux pump activity $\left(P^{<} 0.001\right)$. Additionally, Monk, B. C. et al. and Hayama, K. et al. found that the inhibition of efflux pumps may be involved in the enhanced efficacy of FLC to resistant $C$. albicans and $C$. dubliniensis caused by a D-octapeptide inhibitor of the plasma membrane proton pump (BM2) $)^{16,17}$. These findings demonstrated that the synergism between PPIs and FLC may be related to efflux pumps suppression.

In conclusion, this paper provides an advance over our recent studies and in the field by first finding that PPIs enhanced the efficacy of FLC against resistant C. albicans both in vitro and in vivo and that the efflux pump suppression, extracellular phospholipase inhibition and morphology switching suppression may be involved in the synergistic antifungal effects. These findings together with the opportunity for the combined applications of PPIs and FLC in the clinic will provide new insights into antifungal agent discovery and potential approaches for the treatment of candidiasis caused by resistant C. albicans.

\section{Methods and Materials}

Strains. The six resistant C. albicans strains used in this study are listed in Table 1 . The first three in the table were collected from the clinical laboratory at Shandong Provincial Qianfoshan Hospital (Jinan, China) and the other three were kindly provided by Professor Changzhong Wang (School of Integrated Traditional and Western Medicine, Anhui University of traditional chinese medicine, Hefei, China). C. albicans ATCC10231 was used as a quality control strain to determine the MICs of drugs and was provided by the Institute of Pharmacology, School of Pharmacy, Shandong University (Jinan, China). Strains were stored at $-80^{\circ} \mathrm{C}$ in the Sabouraud dextrose broth and subcultured on the Sabouraud dextrose agar for $24 \mathrm{~h}$ at $35^{\circ} \mathrm{C}$ before the experiment. Egg yolk agar $(0.01 \mathrm{M} \mathrm{NaCl}$, $0.025 \mathrm{M} \mathrm{CaCl}_{2}, 1 \%$ peptone, $3 \%$ glucose, $2 \%$ agar and $10 \%$ egg yolk) was used to test the phospholipase activity.

Drugs. PPIs and FLC were all purchased from Dalian Meilun Biotech Co., Ltd. (Liaoning, China). Stock solutions of OME, PTP, RAB and ESO were prepared in sterile distilled water, while those of FLC, LAN and ILA were prepared in dimethyl sulfoxide. All stock solutions were sterilized using $0.22-\mu \mathrm{m}$ filters, and that of FLC was 
stored at $4{ }^{\circ} \mathrm{C}$ while stock solutions of PPIs were prepared before each experiment. Rhodamine $6 \mathrm{G}$ was purchase from Acros Trading Company.

Antifungal susceptibility testing. The antifungal activities of PPIs and FLC against the six resistant $C$. albicans were tested by the broth microdilution method according to the Clinical and Laboratory Standards Institute (CLSI) guidelines. The test was conducted in 96 -well microtiter plates with yeast $\left(2.5 \times 10^{3} \mathrm{CFU} / \mathrm{mL}\right)$ in RPMI-1640 medium (PH 7.0) buffered with MOPS [morpholino (propanesulfonic acid)]. Wells containing only RPMI-1640 medium served as negative controls, and a drug-free well was set as a growth control. After $24 \mathrm{~h}$ of incubation at $35^{\circ} \mathrm{C}$, the MICs were determined by both visual reading and measuring the optical density (OD) with a microplate reader at a wavelength of $492 \mathrm{~nm}$. The $\mathrm{MIC}_{80}$ was defined as the lowest concentration of drug with $80 \%$ fungal growth inhibition.

Checkerboard microdilution assay. A checkerboard microdilution assay was carried out to determine the interactions between PPIs and FLC against resistant C. albicans. Briefly, drugs were serially diluted 2-fold in RPMI-1640 medium, and 0.25-128 $\mu \mathrm{g} / \mathrm{mL}$ FLC, $2-128 \mu \mathrm{g} / \mathrm{mL}$ OME, LAN, PTP, RAB and ESO and 0.5-32 $\mu \mathrm{g} /$ $\mathrm{mL}$ ILA were added to the wells. Subsequently, yeast at a final concentration of $2.5 \times 10^{3} \mathrm{CFU} / \mathrm{mL}$ was added to each well. Wells containing only RPMI-1640 medium served as negative controls, and a drug-free well was set as a growth control. After $24 \mathrm{~h}$ of incubation at $35^{\circ} \mathrm{C}$, MICs were determined as described above.

To evaluate the mode and intensity of the drug interactions, the fractional inhibitory concentration index (FICI) model and the $\Delta E$ model were used to analyse the obtained data. The FICI model is based on the Loewe additivity theory ${ }^{41}$ and is expressed as $\mathrm{FICI}=\mathrm{FIC}_{\mathrm{A}}+\mathrm{FIC}_{\mathrm{B}}=\frac{\mathrm{MIC}_{\mathrm{A}}^{\text {Comb }}}{\mathrm{MIC}_{\mathrm{A}}^{\text {Alone }}}+\frac{\mathrm{MIC}_{\mathrm{B}}^{\text {Comb }}}{\mathrm{MIC}_{\mathrm{B}}^{\text {Alone }}}$. In this model, the drug interaction is interpreted as synergistic when $\mathrm{FICI} \leq 0.5$, indifferent when $\mathrm{FICl}>0.5-4.0$, and antagonistic when FICI $>4.0$. The $\Delta E$ model is based on the Bliss independence theory $\mathrm{y}^{42}$ and is expressed as $\Delta E=E_{\mathrm{A}} \times E_{\mathrm{B}}-E_{\text {measured }}$. In this equation, $E_{\mathrm{A}}$ and $E_{\mathrm{B}}$ are the experimental fungal growth percentages when each drug acts alone, and $E_{\text {measured }}$ is the measured growth percentage in presence of the combination of drugs A and B. The mode of interaction is interpreted as synergistic when $\Delta E$ and its $95 \%$ confidence interval (CI) are positive, antagonistic when $\Delta E$ and its $95 \% \mathrm{CI}$ are negative, and indifferent in other cases. The intensity of interaction is evaluated by calculating the sum percentages of all significant synergistic $(\Sigma S Y N)$ or antagonistic ( $\Sigma A N T)$ interactions and is interpreted as strong when interactions are $>200 \%$, moderate when interactions are $100-200 \%$, and weak when interactions are $<100 \%$.

Antibiofilm assay. The interactions between PPIs and FLC against preformed biofilms of C. albicans (CA10) in different growth periods were assessed as previously described, with slight modification ${ }^{43}$. Briefly, 200- $\mu \mathrm{L}$ aliquots of a yeast suspension $\left(2.5 \times 10^{3} \mathrm{CFU} / \mathrm{mL}\right)$ were added to a 96 -well plate, and the plates were incubated for four different time intervals $(4,8,12$ and $24 \mathrm{~h})$ at $35^{\circ} \mathrm{C}$ to preform biofilms. Then, the preformed biofilms were washed three times with sterile phosphate-buffered saline (PBS), and drugs were added at final concentrations of $1-1024 \mu \mathrm{g} / \mathrm{mL}$ FLC, $16-1024 \mu \mathrm{g} / \mathrm{mL}$ OME, PTP, RAB and ESO, $8-512 \mu \mathrm{g} / \mathrm{mL}$ LAN and $4-256 \mu \mathrm{g} / \mathrm{mL}$ LAN. Following a further $24 \mathrm{~h}$ of incubation at $35^{\circ} \mathrm{C}$, an XTT reduction assay was performed to examine the metabolic activity of the biofilms. Colorimetric changes were measured with a microplate reader at a wavelength of $492 \mathrm{~nm}$. The sessile minimum inhibitory concentration (sMIC) was defined as the lowest concentration of drug with $80 \%$ inhibition of biofilm metabolic activity.

In vivo infection model. Galleria mellonella larvae were used as an in vivo infection model to evaluate the in vivo interactions between PPIs and FLC, and CA10 was used to infect the G. mellonella larvae ${ }^{44,45}$. For the survival assay, fourteen groups of 20 randomly chosen larvae with a similar size (ca. $0.25 \mathrm{~g}$ ) and no grey markings were selected and injected with $10 \mu \mathrm{L}$ of a yeast suspension $\left(5 \times 10^{8} \mathrm{CFU} / \mathrm{mL}\right)$ via the last left proleg. After $2 \mathrm{~h}$ of incubation at $35^{\circ} \mathrm{C}$, the larvae were injected with $10 \mu \mathrm{L}$ of sterile PBS, $160 \mu \mathrm{g} / \mathrm{mL}$ FLC, PPIs, or PPIs $+160 \mu \mathrm{g} / \mathrm{mL}$ FLC via the last right proleg. The concentration of PPIs was $40 \mu \mathrm{g} / \mathrm{mL}$ RAB and ILA, $80 \mu \mathrm{g} / \mathrm{mL}$ OME, LAN and ESO and $160 \mu \mathrm{g} / \mathrm{mL}$ PTP. Then, the larvae were placed in the dark and incubated at $35^{\circ} \mathrm{C}$ for 4 days. The survival rate of the larvae was monitored daily, considering death of the larvae when they did not respond to physical pressure. For histological study, OME and RAB were selected to evaluate the interactions between PPIs and FLC on the tissue of infected larvae. Seven groups of larvae were selected and injected with a yeast suspension and drugs as described above. One group of larvae untreated with the yeast and drugs served as a blank control group. After a further 48-h incubation, three larvae from each group were randomly selected and cut into sections $(20 \mu \mathrm{m})$. Sections stained with periodic acid Schiff (PAS) stain were observed under a fluorescence microscope.

Yeast-to-hyphae morphogenesis. Effects of PPIs combined with FLC on the yeast-to-hyphae morphogenesis of resistant C. albicans (CA10) were studied in a microplate-based assay ${ }^{46}$. In this assay, OME was selected as the representative PPI, and yeast-to-hyphae morphogenesis was induced by RPMI-1640. Yeast cells $\left(10^{5} \mathrm{CFU} /\right.$ $\mathrm{mL}$ ) were added to a 6-well microplate, and drugs were then added at the final concentration of $8 \mu \mathrm{g} / \mathrm{mL}$ FLC, $128 \mu \mathrm{g} / \mathrm{mL} \mathrm{OME}$ and $64 \mu \mathrm{g} / \mathrm{mL}$ RAB. The microplate was incubated at $35^{\circ} \mathrm{C}$ for $5 \mathrm{~h}$ and then was placed directly under an inverted microscope.

Extracellular phospholipase activity assay. Effects of PPIs combined with FLC on the extracellular phospholipase activity of resistant C. albicans (CA10) were detected by egg yolk agar plates ${ }^{47}$, and OME and RAB were selected as representative PPIs. Yeast cells $\left(10^{6} \mathrm{CFU} / \mathrm{mL}\right)$ were incubated with no drug, FLC $(1 \mu \mathrm{g} / \mathrm{mL}), \mathrm{OME}$ $(32 \mu \mathrm{g} / \mathrm{mL}), \mathrm{RAB}(16 \mu \mathrm{g} / \mathrm{mL})$, OME $(32 \mu \mathrm{g} / \mathrm{mL})$ plus FLC $(1 \mu \mathrm{g} / \mathrm{mL})$, or RAB $(16 \mu \mathrm{g} / \mathrm{mL})$ plus FLC $(1 \mu \mathrm{g} / \mathrm{mL})$ for $24 \mathrm{~h}$ at $35^{\circ} \mathrm{C}$. After the incubation, $10 \mu \mathrm{L}$ of the cell suspensions were inoculated onto egg yolk agar plates and the plates were then incubated for $72 \mathrm{~h}$ at $35^{\circ} \mathrm{C}$. The colony diameter and precipitation zone diameter were measured. 
Efflux pump assay. Whether PPIs interfered with the efflux pump activity of resistant C. albicans was evaluated by the Rh6G efflux assay with some modification ${ }^{48}$, and a resistant isolate with an efflux pump gene over-expressed (CA10) was used. Briefly, yeast cells were incubated in YPD liquid medium overnight, and the cells were harvested, washed with glucose-free PBS and adjusted to $10^{7} \mathrm{CFU} / \mathrm{mL}$. Subsequently, a Rh6G solution was added at a final concentration of $10 \mu \mathrm{M}$ to the cell suspension, and the suspension was incubated at $35^{\circ} \mathrm{C}$ for $50 \mathrm{~min}$ and then exposed to an ice water-bath for $10 \mathrm{~min}$. Cells were harvested and washed with glucose-free PBS. Glucose/PBS (5\%) was added to re-suspend the cells, and PPIs were added. The fluorescence intensity was detected every $60 \mathrm{~min}$ for $4 \mathrm{~h}$ by flow cytometry, with excitation at $488 \mathrm{~nm}$ and emission at $530 \mathrm{~nm}$.

Statistical analysis. Each experiment was performed three times on different days. Graphs were produced with GraphPad Prism 5 and MATLAB 2017a, and statistical analyses were performed with SPSS Statistics v.17.0. The survival curve was analysed by the Kaplan-Meier method and log-rank test. $P$ values $<0.05$ were considered statistically significant.

\section{Data availability}

All data are shown in the paper. Other datasets generated during and/or analyzed during the current study would be provided on reasonable request.

Received: 16 April 2019; Accepted: 4 December 2019;

Published online: 16 January 2020

\section{References}

1. Kullberg, B. J. \& Arendrup, M. C. Invasive Candidiasis. N. Engl. J. Med. 373, 1445-1456 (2015).

2. Cleveland, A. A. et al. Declining incidence of candidemia and the shifting epidemiology of Candida resistance in two US metropolitan areas, 2008-2013: results from population-based surveillance. PLoS one 10, e0120452, https://doi.org/10.1371/journal. pone.0120452 (2015).

3. Polesello, V., Segat, L., Crovella, S. \& Zupin, L. Candida Infections and Human Defensins. Protein Pept. Lett. 24, 747-756 (2017).

4. Azie, N. et al. The PATH (Prospective Antifungal Therapy) Alliance(R) registry and invasive fungal infections: update 2012. Diagn. Microbiol. Infect. Dis. 73, 293-300 (2012).

5. Espinel-Ingroff, A. et al. Multilaboratory study of epidemiological cutoff values for detection of resistance in eight Candida species to fluconazole, posaconazole, and voriconazole. Antimicrob. Agents Chemother. 58, 2006-2012 (2014).

6. Xiao, M. et al. Five-Year National Surveillance of Invasive Candidiasis: Species Distribution and Azole Susceptibility from the China Hospital Invasive Fungal Surveillance Net (CHIF-NET) Study. J. Clin. Microbiol. 56, e00577-18, https://doi.org/10.1128/JCM.00577-18 (2018).

7. Scarpignato, C. et al. Effective and safe proton pump inhibitor therapy in acid-related diseases - A position paper addressing benefits and potential harms of acid suppression. BMC Med. 14, 179 (2016).

8. Malfertheiner, P., Kandulski, A. \& Venerito, M. Proton-pump inhibitors: understanding the complications and risks. Nat. Rev. Gastroenterol. Hepatol. 14, 697-710 (2017).

9. Pereira, O., Figueira-Coelho, J., Picado, B. \& Costa, J. N. Black oesophagus. BMJ Case Rep. 2013, bcr2012008188, https://doi. org/10.1136/bcr-2012-008188 (2013).

10. Chen, K. H., Weng, M. T., Chou, Y. H., Lu, Y. F. \& Hsieh, C. H. Epigastric Distress Caused by Esophageal Candidiasis in 2 Patients Who Received Sorafenib Plus Radiotherapy for Hepatocellular Carcinoma: Case Report. Medicine 95, e3133, https://doi. org/10.1097/MD.0000000000003133 (2016).

11. Shah, A. R., Landsman, M. \& Waghray, N. A Dire Presentation of Diabetic Ketoacidosis with "Black Esophagus. Cureus 11, e4761, https://doi.org/10.7759/cureus.4761 (2019).

12. Biswas, S. K., Yokoyama, K., Kamei, K., Nishimura, K. \& Miyaji, M. Inhibition of hyphal growth of Candida albicans by activated lansoprazole, a novel benzimidazole proton pump inhibitor. Med. Mycol. 39, 283-285 (2001).

13. Liu, N. N. \& Kohler, J. R. Antagonism of Fluconazole and a Proton Pump Inhibitor against Candida albicans. Antimicrob. Agents Chemother. 60, 1145-1147 (2016)

14. Kaneko, Y., Fukazawa, H., Ohno, H. \& Miyazaki, Y. Combinatory effect of fluconazole and FDA-approved drugs against Candida albicans. J. Infect. Chemother. 19, 1141-1145 (2013).

15. Urai, M. et al. Potent drugs that attenuate anti-Candida albicans activity of fluconazole and their possible mechanisms of action. J. Infect. Chemother. 20, 612-615 (2014).

16. Monk, B. C. et al. Surface-active fungicidal D-peptide inhibitors of the plasma membrane proton pump that block azole resistance. Antimicrob. Agents Chemother. 49, 57-70 (2005).

17. Hayama, K. et al. A D-octapeptide drug efflux pump inhibitor acts synergistically with azoles in a murine oral candidiasis infection model. FEMS Microbiol. Lett. 328, 130-137 (2012).

18. Eldesouky, H. E., Mayhoub, A., Hazbun, T. R. \& Seleem, M. N. Reversal of Azole Resistance in Candida albicans by Sulfa Antibacterial Drugs. Antimicrob. Agents Chemother. 62, e00701-17, https://doi.org/10.1128/AAC.00701-17 (2018).

19. Liu, S. et al. Synergistic Effect of Fluconazole and Calcium Channel Blockers against Resistant Candida albicans. PLoS One 11, e0150859, https://doi.org/10.1371/journal.pone.0150859 (2016).

20. Khan, M. S. \& Ahmad, I. Antibiofilm activity of certain phytocompounds and their synergy with fluconazole against Candida albicans biofilms. J. Antimicrob. Chemother. 67, 618-621 (2012).

21. Kim, K. Y. et al. Acid suppression therapy as a risk factor for Candida esophagitis. Dig. Dis. Sci. 58, 1282-1286 (2013).

22. Hasosah, M. Y., Showail, M., Al-Sahafi, A., Satti, M. \& Jacobson, K. Esophageal candidiasis in an immunocompetent girl. World J. Pediatr: WJP 5, 152-154 (2009).

23. Liang, M. et al. A rare case report of fungal esophagitis combined with giant gastric ulcer in an immunocompetent patient. Medicine 98, e14158, https://doi.org/10.1097/MD.0000000000014158 (2019).

24. Mathe, L. \& Van Dijck, P. Recent insights into Candida albicans biofilm resistance mechanisms. Curr. Genet. 59, 251-264 (2013).

25. Cordeiro Rde, A. et al. Exogenous tyrosol inhibits planktonic cells and biofilms of Candida species and enhances their susceptibility to antifungals. FEMS Yeast Res. 15, fov012, https://doi.org/10.1093/femsyr/fov012 (2015).

26. Matsubara, V. H., Wang, Y., Bandara, H. M., Mayer, M. P. \& Samaranayake, L. P. Probiotic lactobacilli inhibit early stages of Candida albicans biofilm development by reducing their growth, cell adhesion, and filamentation. Appl. Microbiol. Biotechnol. 100, 6415-6426 (2016).

27. Velikova, N., Kavanagh, K. \& Wells, J. M. Evaluation of Galleria mellonella larvae for studying the virulence of Streptococcus suis. BMC Microbiol. 16, 291 (2016). 
28. Yang, H. F. et al. Galleria mellonella as an in vivo model for assessing the efficacy of antimicrobial agents against Enterobacter cloacae infection. J. Microbiol. Immunol. Infect. 50, 55-61 (2017).

29. McMillan, S., Verner-Jeffreys, D., Weeks, J., Austin, B. \& Desbois, A. P. Larva of the greater wax moth, Galleria mellonella, is a suitable alternative host for studying virulence of fish pathogenic Vibrio anguillarum. BMC Microbiol. 15, 127 (2015).

30. Scalfaro, C., Iacobino, A., Nardis, C. \& Franciosa, G. Galleria mellonella as an in vivo model for assessing the protective activity of probiotics against gastrointestinal bacterial pathogens. FEMS Microbiol. Lett. 364, fnx064, https://doi.org/10.1093/femsle/fnx064 (2017).

31. Saville, S. P., Lazzell, A. L., Monteagudo, C. \& Lopez-Ribot, J. L. Engineered control of cell morphology in vivo reveals distinct roles for yeast and filamentous forms of Candida albicans during infection. Eukaryot. Cell 2, 1053-1060 (2003).

32. Wilson, D., Naglik, J. R. \& Hube, B. The Missing Link between Candida albicans Hyphal Morphogenesis and Host Cell Damage. PLoS Pathog. 12, e1005867, https://doi.org/10.1371/journal.ppat.1005867 (2016).

33. Martins, M. et al. Morphogenesis control in Candida albicans and Candida dubliniensis through signaling molecules produced by planktonic and biofilm cells. Eukaryot. Cell 6, 2429-2436 (2007).

34. Noble, S. M., French, S., Kohn, L. A., Chen, V. \& Johnson, A. D. Systematic screens of a Candida albicans homozygous deletion library decouple morphogenetic switching and pathogenicity. Nat. Genet. 42, 590-598 (2010).

35. Ibrahim, A. S. et al. Evidence implicating phospholipase as a virulence factor of Candida albicans. Infect. Immun. 63, 1993-1998 (1995).

36. Ghannoum, M. A. Potential role of phospholipases in virulence and fungal pathogenesis. Clin. Microbiol. Rev. 13, 122-143 (2000).

37. Ying, S. \& Chunyang, L. Correlation between phospholipase of Candida albicans and resistance to fluconazole. Mycoses 55, 50-55 (2012).

38. Hube, B. et al. The role and relevance of phospholipase D1 during growth and dimorphism of Candida albicans. Microbiology 147, 879-889 (2001).

39. Prasad, R. \& Rawal, M. K. Efflux pump proteins in antifungal resistance. Front. pharmacol. 5, 202, https://doi.org/10.3389/ fphar.2014.00202 (2014).

40. Prasad, R. \& Goffeau, A. Yeast ATP-binding cassette transporters conferring multidrug resistance. Annu. Rev. Microbiol. 66, 39-63 (2012).

41. Odds, F. C. Synergy, antagonism, and what the chequerboard puts between them. J. Antimicrob. Chemother. 52, 1, https://doi. org/10.1093/jac/dkg301 (2003).

42. Katragkou, A. et al. In vitro interactions between farnesol and fluconazole, amphotericin B or micafungin against Candida albicans biofilms. J. Antimicrob. Chemother. 70, 470-478 (2015).

43. Ramage, G. \& Lopez-Ribot, J. L. Techniques for antifungal susceptibility testing of Candida albicans biofilms. Methods Mol. Med. 118, 71-79 (2005).

44. Gu, W., Yu, Q., Yu, C. \& Sun, S. In vivo activity of fluconazole/tetracycline combinations in Galleria mellonella with resistant Candida albicans. infection. Glob. Antimicrob. Resist. 13, 74-80 (2017).

45. Gu, W., Guo, D., Zhang, L., Xu, D. \& Sun, S. The Synergistic Effect of Azoles and Fluoxetine against Resistant Candida albicans Strains Is Attributed to Attenuating Fungal Virulence. Antimicrob. Agents Chemother. 60, 6179-6188 (2016).

46. Zhong, H. et al. Activity of Sanguinarine against Candida albicans Biofilms. Antimicrob. Agents Chemother. 61, e02259-16, https:// doi.org/10.1128/AAC.02259-16 (2017).

47. Shirkhani, S., Sepahvand, A., Mirzaee, M. \& Anbari, K. Phospholipase and proteinase activities of Candida spp. isolates from vulvovaginitis in Iran. J. Mycol. Med. 26, 255-260 (2016).

48. Sharma, M. \& Prasad, R. The quorum-sensing molecule farnesol is a modulator of drug efflux mediated by ABC multidrug transporters and synergizes with drugs in Candida albicans. Antimicrob. Agents Chemother. 55, 4834-4843 (2011).

\section{Acknowledgements}

We are very grateful for the administration of traditional Chinese Medicine of Shandong Province, China [2017166] and the financial support from the Department of Health of Shandong Province, China [2016GSF201187], and we wish to acknowledge all members who contributed to the antifungal resistance study group.

\section{Author contributions}

M.J.L. and S.J.S. conceived and designed the experiments; M.J.L. performed the experiments; M.J.L., H.Y.Y., C.X.Y., L.Y. and S.J.S. analyzed the data; H.Y.Y., C.X.Y. and S.J.S. contributed reagents/materials/analysis tools; M.J.L., L.Y. and S.J.S. wrote the paper. All authors read and approved the final manuscript.

\section{Competing interests}

The authors declare no competing interests.

\section{Additional information}

Supplementary information is available for this paper at https://doi.org/10.1038/s41598-019-57174-4.

Correspondence and requests for materials should be addressed to S.S.

Reprints and permissions information is available at www.nature.com/reprints.

Publisher's note Springer Nature remains neutral with regard to jurisdictional claims in published maps and institutional affiliations.

(c) (i) Open Access This article is licensed under a Creative Commons Attribution 4.0 International

License, which permits use, sharing, adaptation, distribution and reproduction in any medium or format, as long as you give appropriate credit to the original author(s) and the source, provide a link to the Creative Commons license, and indicate if changes were made. The images or other third party material in this article are included in the article's Creative Commons license, unless indicated otherwise in a credit line to the material. If material is not included in the article's Creative Commons license and your intended use is not permitted by statutory regulation or exceeds the permitted use, you will need to obtain permission directly from the copyright holder. To view a copy of this license, visit http://creativecommons.org/licenses/by/4.0/.

(c) The Author(s) 2020 